\title{
A three dimensional magnetohydrodynamic pulse in a transversely inhomogeneous medium
}

\author{
D. Tsiklauri and V. M. Nakariakov
}

\author{
Physics Department, University of Warwick, Coventry, CV4 7AL, UK
}

Received 25 February 2002 / Accepted 11 July 2002

\begin{abstract}
Interaction of impulsively generated MHD waves with a one-dimensional plasma inhomogeneity, transverse to the magnetic field, is considered in the three-dimensional regime. Because of the transverse inhomogeneity, MHD fluctuations, even if they do not include initially any density perturbation, evolve toward states where the compressible components tend to become predominant. The propagating MHD pulse asymptotically reaches a quasi-steady state with the final levels of density perturbation weakly depending on the degree of non-planeness of the pulse in the homogeneous transverse direction and somewhat stronger depending on plasma $\beta$. Our study demonstrates the necessity of incorporation of compressible and 3D effects in theory of Alfvén wave phase mixing. However, as far as the dynamics of weakly non-plane Alfvén waves is concerned it can still be qualitatively understood in terms of the previous $2.5 \mathrm{D}$ models.
\end{abstract}

Key words. magnetohydrodynamics (MHD) - waves - Sun: activity - Sun: solar wind

\section{Introduction}

Problems of heating of the open corona of the Sun and acceleration of the solar wind are closely related with the interaction of MHD waves with plasma inhomogeneities and, in particular, with coupling of compressible and incompressible components of the waves. Investigation of coupling mechanisms between the waves is of prime importance because, on one hand, the Alfvén waves are usual candidates of energy transport from the lower layers of the solar atmosphere to the corona, and, on the other, compressible perturbations are subject to much more efficient dissipation than the incompressible Alfvén waves (see, e.g., Narain \& Ulmschneider 1996). Also, the compressive waves, as opposed to the Alfvén waves, can transport energy across the magnetic field, due to the fact that their propagation in space is not constrained by the field lines.

The original idea of the phase mixing of incompressible Alfvén waves (Heyvaerts \& Priest 1983) was based on the following argument: when plasma has a density gradient perpendicular to the magnetic field, local Alfvén speed is a function of the transverse coordinate. Thus, when an Alfvén wave propagates along the field its perturbations on the adjacent field lines become out of phase. This stretching of Alfvén wave front creates progressively smaller spatial scales across the field. In turn, because the dissipation is proportional to the wave number squared, phase mixing leads to enhanced dissipation of the Alfvén wave directly. In the compressible plasma, as demonstrated by Malara et al. (1996), Nakariakov et al. (1997),

Send offprint requests to: D. Tsiklauri,

e-mail: tsikd@astro.warwick.ac.uk
Nakariakov et al. (1998), Botha et al. (2000), Tsiklauri et al. (2001), Tsiklauri et al. (2002), phase mixing of linearly polarized plane Alfvén waves leads to the enhanced nonlinear generation of fast magnetoacoustic waves. However, it was established, in 2.5D geometry, for the harmonic Alfvén wave by Botha et al. (2000), and for a wide spectrum Alfvén pulse by Tsiklauri et al. (2001) that compressive perturbations, which are initially absent from the system, do not grow to a substantial fraction of the initial Alfvén wave due the destructive wave interference effect.

In this work we consider fully three dimensional geometry and study interaction of linear (with the non-linear effects totally ignored) MHD waves with a one-dimensional inhomogeneity of the plasma, taking into account compressibility of the plasma and the localization of the MHD pulse in the direction perpendicular to both the magnetic field and the inhomogeneity gradient. In particular, we shall study how the phenomenon of phase mixing is affected by these factors. A similar problem was studied by De Groof \& Goossens (2000) in the context of resonant absorption of MHD waves in coronal loops as a heating mechanism. However, our treatment is more general as we perform a direct numerical 3D simulation without resorting to Fourier transform in time and $y$ direction (and, consequently, our study can easily be generalized to the case of 2D and 3D structuring) allowing us to consider an initial value problem. Besides, De Groof \& Goossens (2000) used a harmonic form of the initial perturbation as opposed to our spatially localized, Gaussian, one. Recently, Hood et al. (2002) showed that the wide spectrum regime of phase mixing can be quite different from the harmonic one, as in fact, phase 
mixing of localized Alfvén pulses results in a slower, algebraic, damping as opposed to the standard exponential damping of harmonic Alfvén waves, which suggest that localized Alfvénic perturbations will transport energy higher into the corona than harmonic ones. Such Alfvénic perturbations could be generated e.g. by transient events such as solar flares, coronal mass ejections, etc. While most of the phase-mixing studies concentrated on harmonic perturbations (e.g. Heyvaerts \& Priest 1983; Hood et al. 1997; Hood et al. 1997; Nakariakov et al. 1998; Ruderman et al. 1998; DeMoortel et al. 1999; DeMoortel et al. 2000; Grappin et al. 2000; Botha et al. 2000), only few considered spatially localized ones (Nakariakov et al. 1997; Tsiklauri et al. 2001; Tsiklauri et al. 2002; Hood et al. 2002).

An additional motivation to this study is connected with the growing interest to the problem of interaction of MHD waves with $2 \mathrm{D}$ and 3D plasma structures and irregularities, as the coronal and wind plasmas are observed to be structured in all three dimensions. 2D and 3D structuring can dramatically affect properties of MHD waves. In particular, as it was shown in the incompressible regime by Similon \& Sudan (1989) and confirmed in numerical experiments performed by Petkaki et al. (1998) and Malara et al. (2000), the structuring dramatically increases the efficiency of wave dissipation. However, in those studies, the compressible effects were not taken into account. From this point of view, our investigation of the interaction of a 3D MHD pulse with a 1D plasma inhomogeneity in the compressible regime provides a necessary building element of the general theory of MHD wave interaction with plasma inhomogeneities.

The present model is based upon the MHD description of plasma and we focus on the problems that are of relevance to the heating of solar corona, acceleration and the dynamics of solar wind. While, similar studies exist that deal with, for instance, terrestrial auroral applications. Namely, an alternative to MHD, more relevant, particle-in-cell simulations (using a guiding center implicit code for the electrons) have been performed (Génot et al. 1999; Génot et al. 2000; Génot et al. 2001), which study the propagation and collisionless dissipation (via effective electron beam generation) of the Alfvén waves in the auroral density inhomogeneities (cavities).

In this work we study the propagation part of the problem, i.e. we consider an ideal plasma limit. The work is in progress to include finite plasma resistivity in order to investigate quantitatively the dependence of the decay of Alfvénic part of the MHD pulse upon the coupling to the existing compressive waves.

The paper is organized as follows: in Sect. 2 we formulate our model. In Sect. 3 we present the results of numerical simulation, while we close in Sect. 4 with the discussion of main results.

\section{The model}

In our model we use equations ideal MHD

$$
\begin{aligned}
& \rho \frac{\partial \boldsymbol{V}}{\partial t}+\rho(\boldsymbol{V} \cdot \nabla) \boldsymbol{V}=-\nabla p-\frac{1}{4 \pi} \boldsymbol{B} \times \operatorname{curl} \boldsymbol{B}, \\
& \frac{\partial \boldsymbol{B}}{\partial t}=\operatorname{curl}(\boldsymbol{V} \times \boldsymbol{B}),
\end{aligned}
$$

$$
\frac{\partial p}{\partial t}+\boldsymbol{V} \cdot \nabla p+\gamma p \nabla \cdot \boldsymbol{V}=0
$$

where $\boldsymbol{B}$ is the magnetic field, $\boldsymbol{V}$ is plasma velocity, $\rho$ is plasma mass density, and $p$ is plasma thermal pressure. In what follows we use $5 / 3$ for the value of $\gamma$.

We solve Eqs. (1)-(3) in Cartesian coordinates $(x, y, z)$. Note that as we solve a fully $3 \mathrm{D}$ problem we retain variation in the $y$-direction, i.e. $(\partial / \partial y \neq 0)$. The equilibrium state is taken to be an inhomogeneous plasma of density $\rho_{0}(x)$ and a uniform magnetic field $B_{0}$ in the $z$-direction. We consider a plasma configuration similar to the one investigated in Malara et al. (1996), Nakariakov et al. (1997), Nakariakov et al. (1998), Botha et al. (2000), Tsiklauri et al. (2001), i.e. the plasma has a one-dimensional inhomogeneity in the equilibrium density $\rho_{0}(x)$ and temperature $T_{0}(x)$. The unperturbed thermal pressure, $p_{0}$, is taken to be constant everywhere.

Next, we do usual linearization of the Eqs. (1)-(3) and write them in component form as following

$$
\begin{aligned}
& \rho_{0}(x) \frac{\partial V_{x}}{\partial t}+\frac{\partial p}{\partial x}-\frac{B_{0}}{4 \pi}\left(\frac{\partial B_{x}}{\partial z}-\frac{\partial B_{z}}{\partial x}\right)=0, \\
& \rho_{0}(x) \frac{\partial V_{y}}{\partial t}+\frac{\partial p}{\partial y}+\frac{B_{0}}{4 \pi}\left(\frac{\partial B_{z}}{\partial y}-\frac{\partial B_{y}}{\partial z}\right)=0, \\
& \rho_{0}(x) \frac{\partial V_{z}}{\partial t}+\frac{\partial p}{\partial z}=0 \\
& \frac{\partial B_{x}}{\partial t}-B_{0} \frac{\partial V_{x}}{\partial z}=0, \\
& \frac{\partial B_{y}}{\partial t}-B_{0} \frac{\partial V_{y}}{\partial z}=0, \\
& \frac{\partial B_{z}}{\partial t}+B_{0}\left(\frac{\partial V_{x}}{\partial x}+\frac{\partial V_{y}}{\partial y}\right)=0, \\
& \frac{\partial p}{\partial t}+\gamma p_{0}\left(\frac{\partial V_{x}}{\partial x}+\frac{\partial V_{y}}{\partial y}+\frac{\partial V_{z}}{\partial z}\right)=0 .
\end{aligned}
$$

It is useful to re-write Eqs. (4)-(10) in a form of three coupled wave equations as following

$$
\begin{gathered}
{\left[\partial_{t t}^{2}-\left(c_{\mathrm{s}}^{2}(x)+c_{\mathrm{A}}^{2}(x)\right) \partial_{x x}^{2}-c_{\mathrm{A}}^{2}(x) \partial_{z z}^{2}\right] V_{x}} \\
-\left[\left(c_{\mathrm{s}}^{2}(x)+c_{\mathrm{A}}^{2}(x)\right) \partial_{x y}^{2}\right] V_{y}-\left[c_{\mathrm{s}}^{2}(x) \partial_{x z}^{2}\right] V_{z}=0 \\
{\left[\partial_{t t}^{2}-\left(c_{\mathrm{s}}^{2}(x)+c_{\mathrm{A}}^{2}(x)\right) \partial_{y y}^{2}-c_{\mathrm{A}}^{2}(x) \partial_{z z}^{2}\right] V_{y}} \\
-\left[\left(c_{\mathrm{s}}^{2}(x)+c_{\mathrm{A}}^{2}(x)\right) \partial_{x y}^{2}\right] V_{x}-\left[c_{\mathrm{s}}^{2}(x) \partial_{y z}^{2}\right] V_{z}=0 \\
{\left[\partial_{t t}^{2}-c_{\mathrm{s}}^{2}(x) \partial_{z z}^{2}\right] V_{z}-\left[c_{\mathrm{s}}^{2}(x) \partial_{x z}^{2}\right] V_{x}-\left[c_{\mathrm{s}}^{2}(x) \partial_{y z}^{2}\right] V_{y}=0}
\end{gathered}
$$

where $c_{\mathrm{A}}(x)=B_{0} / \sqrt{4 \pi \rho_{0}(x)}$ and $c_{\mathrm{s}}(x)=\sqrt{\gamma p_{0} / \rho_{0}(x)}$ denote local Alfvén and sound speeds respectively.

We solve Eqs. (4)-(10) numerically after re-writing them in a dimensionless form using following normalization: $B_{x, y, z}=B_{0} \bar{B}_{x, y, z},(x, y, z)=a_{*}(\bar{x}, \bar{y}, \bar{z}), c_{\mathrm{A}}(x)=B_{0} / \sqrt{4 \pi \rho_{0}(x)}=$ $B_{0} / \sqrt{4 \pi \rho_{*}} / \sqrt{3-2 \tanh (\lambda x)}=c_{\mathrm{A}}^{*} / \sqrt{3-2 \tanh (\lambda x)}, t=$ $\left(a_{*} / c_{\mathrm{A}}^{*}\right) \bar{t}, V_{x, y, z}=c_{\mathrm{A}}^{*} \bar{V}_{x, y, z}$. Note, that $c_{\mathrm{s}}(x)=\sqrt{\gamma \beta / 2} c_{\mathrm{A}}(x)$, where $\beta$ stands for the ratio of thermal and magnetic pressures $\beta=p_{0} /\left(B_{0}^{2} / 8 \pi\right)$. Here, $\lambda$ is a free parameter which controls the steepness of the density profile gradient. In our simulations we use $\lambda=0.5$. In what follows we omit bars on top of the physical quantities. 

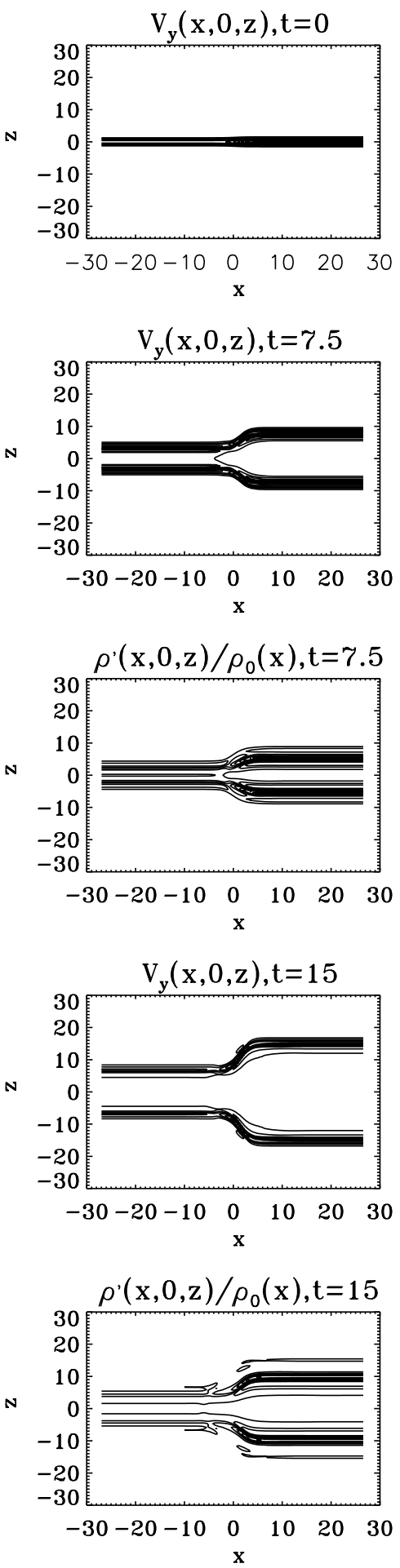
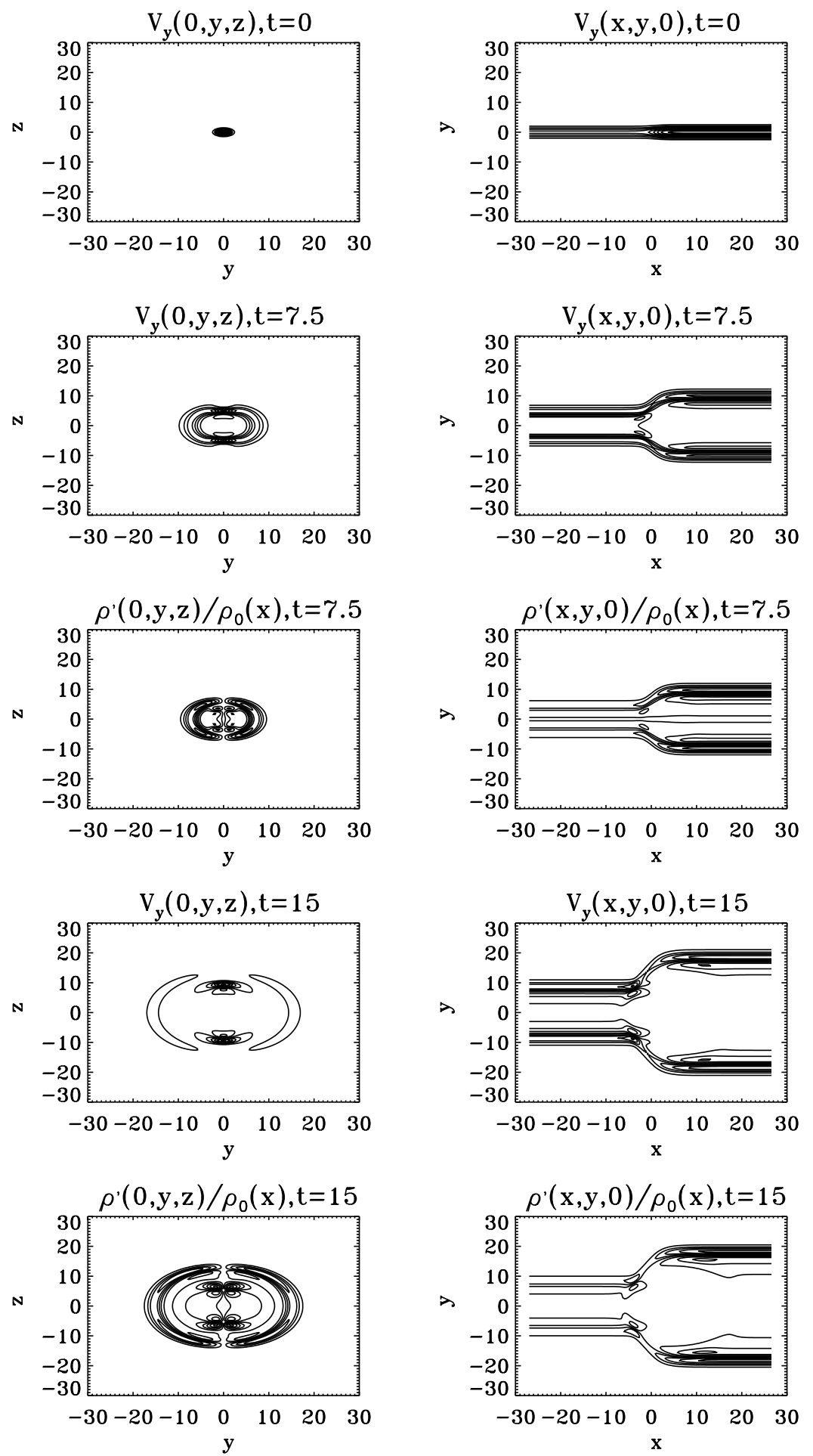

Fig. 1. Contour plots of $V_{y}$ and $\rho^{\prime} / \rho_{0}(x)$ (relative density perturbation) through three cross-sections at the same time snapshots. Here, $\beta=0.5$, $\alpha_{y}=0.6, \alpha_{z}=1.0$.

\section{Numerical results}

In order to solve Eqs. (4)-(10) numerically we have written a new numerical code $d t 4 d x 10$, which uses a high order finite difference scheme. Namely, it evaluates 10th order centered spatial derivatives, and advances solution in time using 4th order Runge-Kutta algorithm. Therefore, $d t 4 d x 10$ is $O\left[(\Delta t / T)^{4}\right]$ accurate in time and $O\left[(\max (\Delta x, \Delta y, \Delta z) / L)^{10}\right]$ accurate in space, with $T$ and $L$ denoting run time and linear size of the simulation domain. The use of such a high-order numerical approach was motivated by the very nature of the problem considered, as development of phase mixing leads to the generation of very steep profiles in the wave front. 


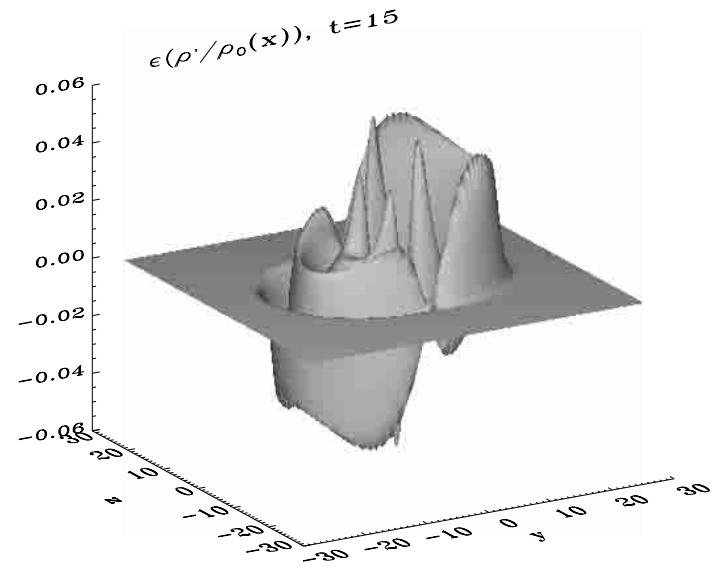

Fig. 2. A snapshot of relative perturbation of plasma density by an MHD pulse interacting with a one-dimensional inhomogeneity at $t=15$ through $x=0$ cross-section. Here, $\beta=0.5, \alpha_{y}=0.6, \alpha_{z}=1.0$.

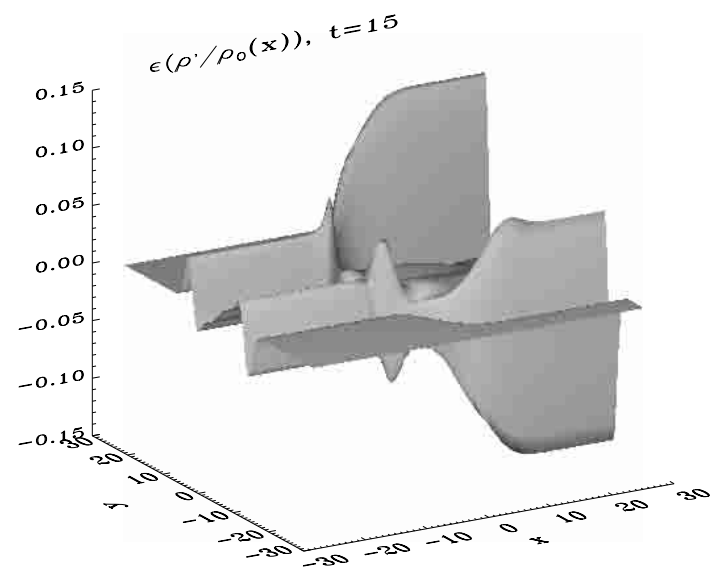

Fig. 3. The same as in Fig. 2, but through $z=0$ cross-section.

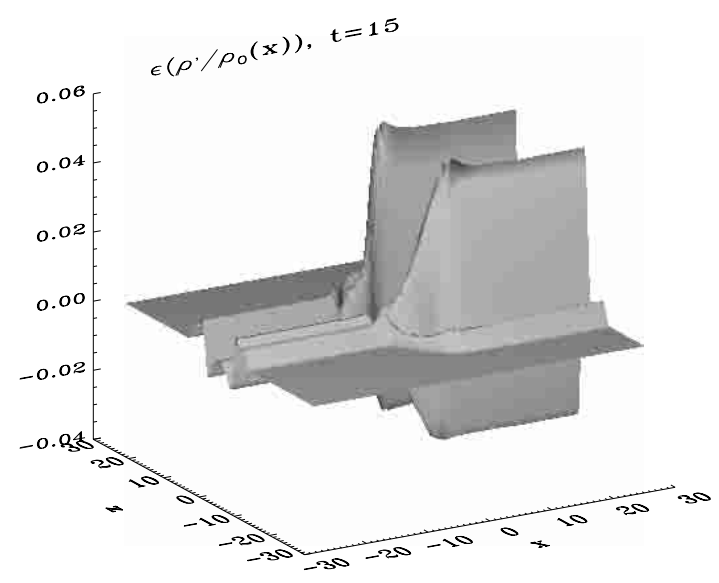

Fig. 4. The same as in Fig. 2, but through $y=0$ cross-section.

The simulation cube size is set by the limits $-25.0 \leq$ $x \leq 25.0,-25.0 \leq y \leq 25.0$ and $-25.0 \leq z \leq 25.0$. Boundary conditions used in all our simulations are zero-gradient in all three spatial dimensions.

We have performed calculation on various resolutions in attempt to achieve convergence of the results. The graphical results presented here are for the spatial resolution $128^{3}$, which refers to number of grid points in $x, y$ and $z$ directions respectively. We have also performed calculation on the spatial resolution $256^{3}$ and we found that the results converge perfectly. This is understandable due to the high order of the scheme even for the $128^{3}$ resolution time-error, $O\left[(\Delta t / T)^{4}\right] \approx 7 \times 10^{-8}$, and spatial-error, $O\left[(\max (\Delta x, \Delta y, \Delta z) / L)^{10}\right] \approx 8 \times 10^{-22}$. Since a $256^{3}$ resolution run takes about 8 hours on Compaq ES40 with eight EV6 500-MHz processors, while a $128^{3}$ resolution takes only $1 \mathrm{~h}$ on Compaq ES40 with four EV6 500-MHz processors producing the same results, we opted for the latter, less CPU-consuming, alternative.

In the numerical simulations the MHD perturbation is initially a plane (with respect to $x$-coordinate) pulse, which has a Gaussian structure in $y$ and $z$-coordinates

$V_{y}(x, y, z, t=0)=c_{\mathrm{A}}(x) \exp \left(-\left(\alpha_{y} y\right)^{2}-\left(\alpha_{z} z\right)^{2}\right)$.

Here, $\alpha_{y}$ and $\alpha_{z}$ are free parameters which control the strength of gradients in $y$ and $z$ direction of the initial perturbation. As the problem considered is linear, the wave amplitude can be taken to be normalized to unity.

In the geometry considered, when the initial perturbation depends on $y$ it cannot be regarded as pure Alfvénic one. In fact, in such a pulse, all three waves - Alfvén, fast and slow magnetosonic waves - are inter-coupled so that there is no use of their separation per se. The pulse is set to be initially plane in the $x$-direction. This allows us to emphasize the effect of the inhomogeneity on the pulse evolution. From the point of view of applications, this simply means that the initial characteristic size of the pulse in that direction is greater than the scale of the inhomogeneity.

The particular choice of the initial condition Eq. (14) is motivated by the argument that when we set $B_{y}$ initially to zero we automatically guarantee fulfillment of $\operatorname{div} \boldsymbol{B}=0$ when $\alpha_{y} \neq 0$, and as the system evolves it adjusts itself which mode to excite (depending whether $\alpha_{y}$ is zero or not). For instance, if we choose our initial conditions as $V_{y}$ given by Eq. (14), $B_{y}(x, y, z, t=0)=\exp \left(-\left(\alpha_{y} y\right)^{2}-\left(\alpha_{z} z\right)^{2}\right)$, with $\alpha_{y}=0$ and the rest of physical quantities set to zero, we would excite a pure Alfvénic pulse traveling in the negative direction along $z$-axis. However, as long as $\alpha_{y} \neq 0$ we have to set $B_{y}(x, y, z, t=0)=0$ in order to fulfill $\operatorname{div} \boldsymbol{B}=0$. Besides, our choice of the localized "kinematic" (the velocity is initially perturbed only, while the magnetic field is constant) perturbation Eq. (14) is well motivated by the fact that such perturbations can arise, e.g., during coronal mass ejections, solar flares, or other violent events (see, e.g., Roussev et al. 2001), etc.

In Fig. 1 we present time evolution of the transverse component of the plasma velocity, $V_{y}$ and the density perturbation $\rho^{\prime} / \rho_{0}(x)$. The choice of these physical quantities is motivated by the following reasoning: the transverse velocity component $V_{y}$ is subject to Alfvén wave phase mixing and the density perturbation demonstrates the effect of the plasma compressibility. The left column of Fig. 1 shows three snapshots of $V_{y}$ and two snapshots $\left(\rho^{\prime} / \rho_{0}(x)\right)$ through the $y=0$ cross-section, the middle column shows three snapshots of $V_{y}$ and two snapshots $\left(\rho^{\prime} / \rho_{0}(x)\right)$ through the $x=0$ cross-section, and the right column shows three snapshots of $V_{y}$ and two snapshots $\left(\rho^{\prime} / \rho_{0}(x)\right)$ 

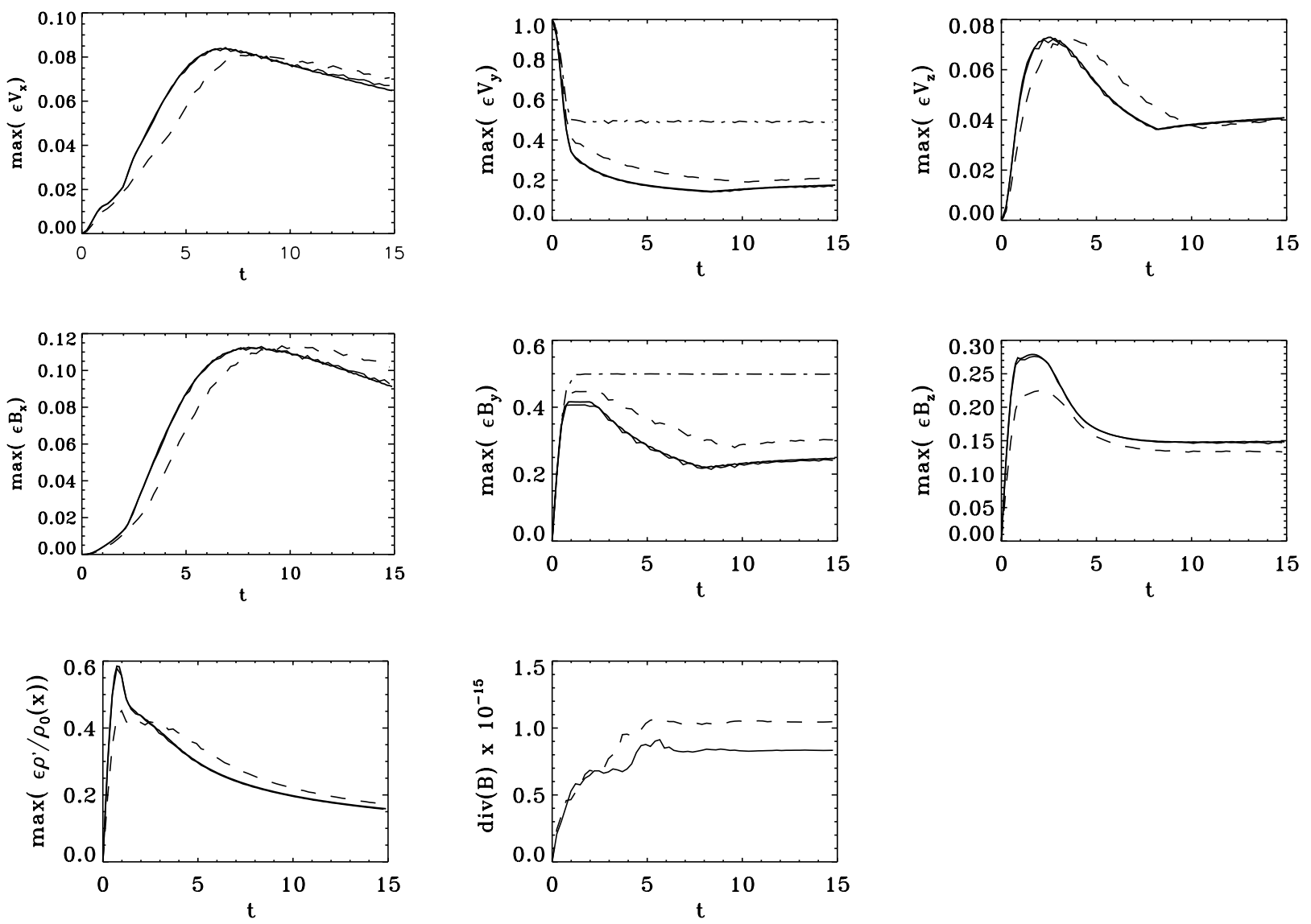

Fig. 5. Maximum of the absolute values over the whole 3D simulation box of all physical quantities as a function of time. Here, $\beta=0.5$ and $\alpha_{z}=1.0$. Thick solid lines correspond to the resolution $256^{3}$, while thin solid lines to that of $128^{3}$, both for $\alpha_{y}=0.6$. Note that on all seven panels these two curves practically overlap, which serves as a proof of convergence of our simulation results. Dashed lines represent $\alpha_{y}=0.4$, while dash-dotted lines show $\alpha_{y}=0$.
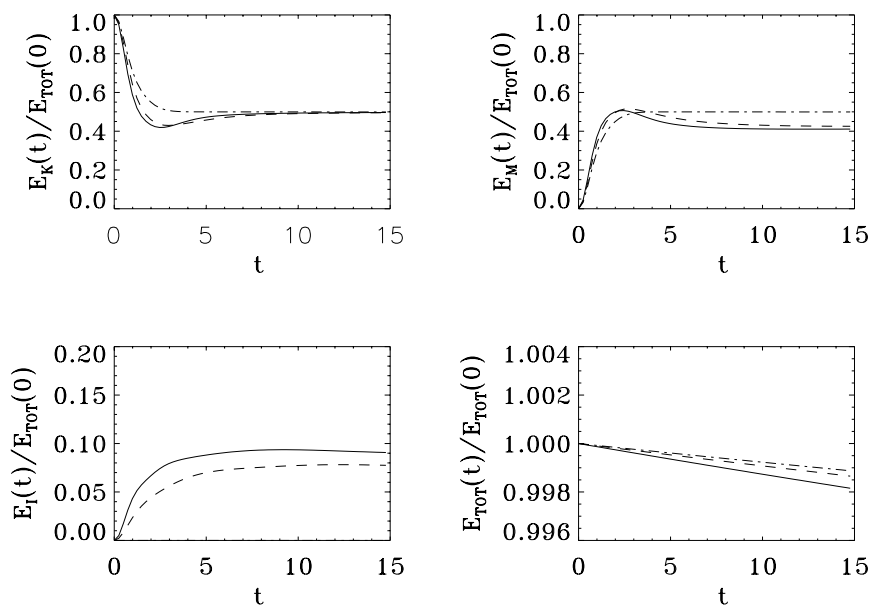

Fig. 6. Normalized kinetic, magnetic, internal and total energies (all of them integrated over the volume) as a function of time. Here, $\beta=0.5$ and $\alpha_{z}=1.0$. Solid lines correspond to $\alpha_{y}=0.6$, while dashed and dash-dotted lines show cases of $\alpha_{y}=0.4$ and $\alpha_{y}=0$ respectively.

through the $z=0$ cross-section. The snapshots of $V_{y}$ and $\rho^{\prime} / \rho_{0}(x)$ at times $t=7.5$ and 15 are placed such that it is easier to spot the differences. The snapshot of $\rho^{\prime} / \rho_{0}(x)$ at $t=0$ is not present in the figure as it is identically zero and it is generated later by the $y$-gradients in the function $V_{y}$. This compressible perturbation is a significant component of the pulse. What we gather from this graph is as following:

$-y=0$ cross-section: the initial MHD pulse, which is plane with respect to $x$-coordinate, is split in two D'Alambert's solutions, with half of the amplitudes, traveling in two opposite directions along the magnetic field. Because the unperturbed density is inhomogeneous across the magnetic field (in the $x$-direction) the local Alfvén speed depends upon $x$. Thus, the Alfvénic pulse is phase-mixed and its initially plane front is continuously distorted creating transverse gradients. Note, that the right wing of the pulse is asymmetric to the left one, this is due to the fact that its amplitude drops from 1.0 in the $x>0$ domain to $1 / \sqrt{5}$ in the $x<0$. The generated density perturbation evolves in the similar manner, however there is a notable difference - as plasma $\beta=0.5$ in this case, we observe that the density perturbation travels at a slower speed (slow magnetosonic wave speed) than the Alfvénic one. If we look at the right wing of $V_{y}(x, 0, z)$ at $t=15$, we see that it traveled to $z=15$, which is consistent with the Alfvén speed equals 1 (recall that for $x>0 c_{\mathrm{A}}(x)=1$ ). The density perturbation as it travels along the field should have a velocity of a slow magnetosonic wave, which for the field aligned propagation 

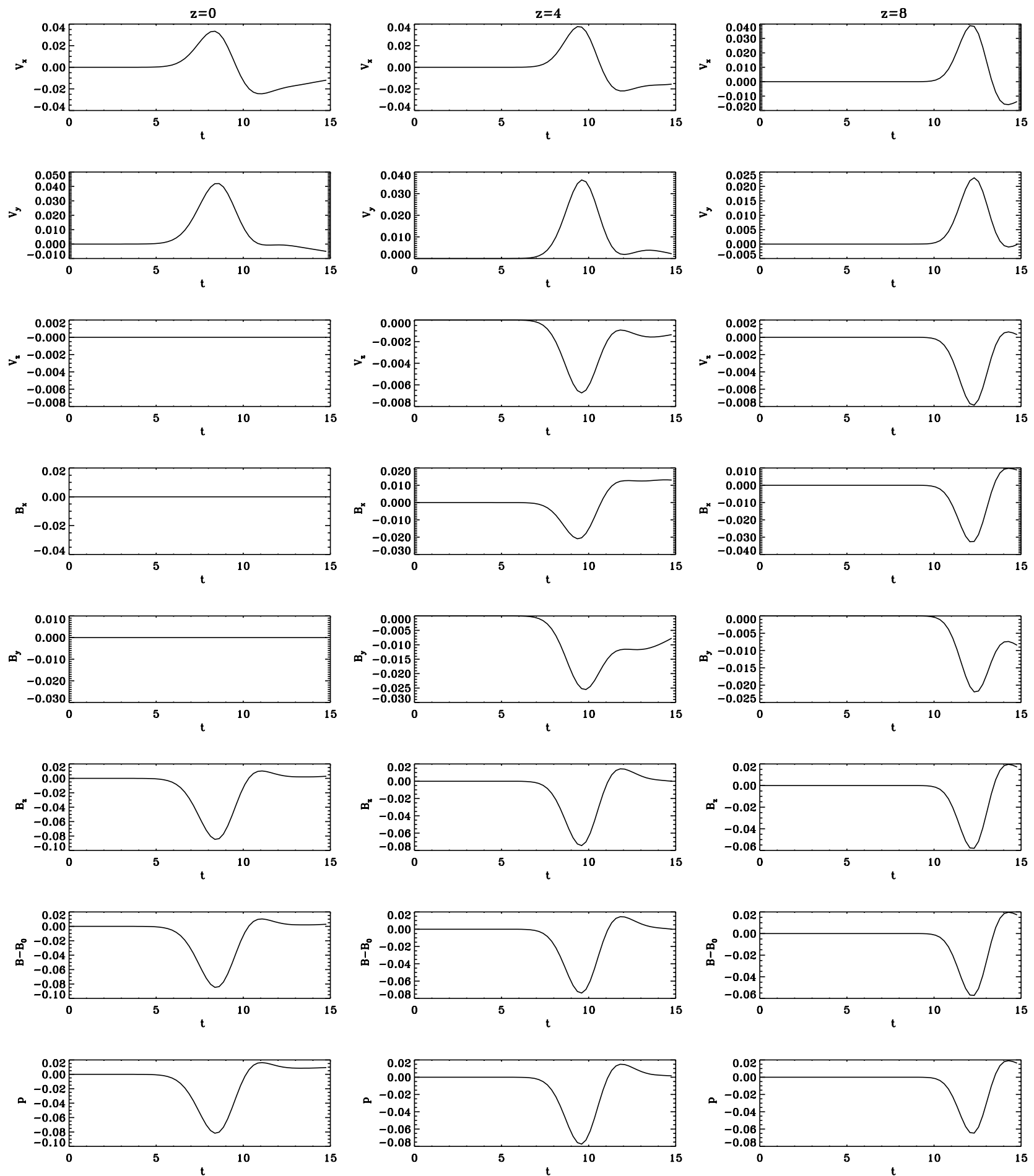

Fig. 7. All physical quantities perturbed by the MHD pulse as a function of time at a given point in the phase mixing region. Here, $\beta=0.5$, $\alpha_{y}=0.6, \alpha_{z}=1.0$. Left column: at the point $(x=0, y=-8, z=0)$, mid column: at the point $(x=0, y=-8, z=4)$, right column at the point $(x=0, y=-8, z=8)$.

coincides with the local speed of sound. For the parameters considered $c_{\mathrm{s}}(x)=\sqrt{\gamma \beta / 2} c_{\mathrm{A}}(x)=0.65 c_{\mathrm{A}}(x)$. Therefore, the right wing of the density perturbation at $t=15$ should have traveled to a position $z \approx 9.7$. That is what we actually see from the bottom panel of the left column in Fig. 1;

- $x=0$ cross-section: the initial Alfvénic pulse which has an asymmetry as $\alpha_{y} \neq \alpha_{z}$ Gaussian bell shape is split in two smaller amplitude bells traveling in two opposite directions along the magnetic field plus some semielliptic shaped wake expanding outwards in all directions. Note, that because the unperturbed density is homogeneous across the magnetic field in $y$-direction we do not see any distortions on the wave front with respect to the $y=0$ line as we saw in the previous case. 

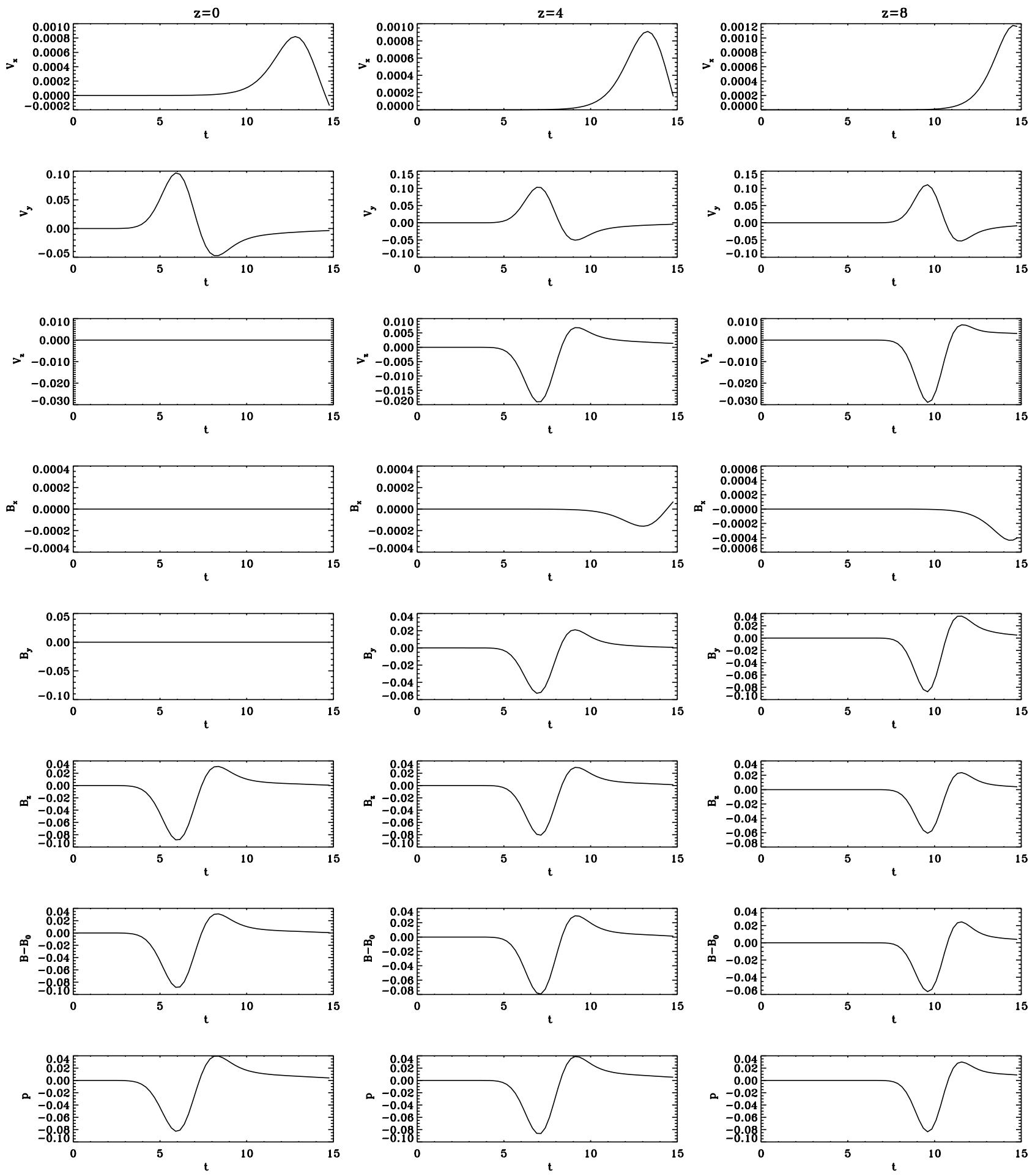

Fig. 8. All physical quantities as a function of time at a given point far from the phase mixing region. Here, $\beta=0.5, \alpha_{y}=0.6, \alpha_{z}=1.0$. Left column: at the point $(x=16, y=-8, z=0)$, mid column: at the point $(x=16, y=-8, z=4)$, right column at the point $(x=16, y=-8$, $z=8)$.

The generated density perturbation evolves in the similar manner, however a notable difference (can be better seen from Fig. 2) is that the solution consists of positive and negative spikes plus semi-elliptic shaped wakes that are symmetric with respect to $\rho^{\prime} / \rho_{0}(x)=0$ plane.
This can be understood readily as due to the conservation of total mass, integral of density perturbation over the volume should, indeed, be zero;

$-z=0$ cross-section: the MHD pulse evolution, viewed through this cross-section, is similar to the case of $y=0$ 
cross-section (see above). Again we see two phase-mixed D'Alambert's solutions propagating in the opposite directions. The notable difference is that through $z=0$ crosssection the pulse looks wider as $\alpha_{y}=0.6$ while $\alpha_{z}=1.0$. The generated density perturbation evolves in the similar manner, as the Alfvénic one. However, in differ from $y=0$ cross-section, we see no difference in traveling speeds. This can be explained as following: the pulse was not excited as a "pure Alfvén wave". In fact, should the perturbation $V_{y}$ be purely Alfvénic there would have been no propagation along $y$ axis at all (recall that the magnetic field is directed along the $z$-axis). Thus, what we see through the $z=0$ cross-section both for $V_{y}$ and $\rho^{\prime} / \rho_{0}(x)$ is the compressive part of the perturbation traveling at the fast magnetosonic wave speed. For the parameters considered, the fast magnetosonic wave speed is $\sqrt{1+\gamma \beta / 2} c_{\mathrm{A}}(x)=1.2 c_{\mathrm{A}}(x)$. Therefore, the right wing of both $V_{y}$ and density perturbation at $t=15$ should have traveled to a position $y \approx 17.9$. That is what we actually observe from the two bottom panels of the right column in Fig. 1.

Also, the figure shows the obvious anisotropy in the pulse propagation, connected with the presence of two chosen directions: the direction of the straight magnetic field and the direction of the density gradient.

Perturbation of $B_{y}$ is initially absent from the system, but as it is a potential component of the kinetic counterpart, $V_{y}$, of the wave, it is soon generated and further evolves similarly to $V_{y}$ in a form of two negative and positive phase-mixed pulses traveling into two opposite directions. Also, the development of the pulse is accompanied by generation of compressible components. The structure of the density perturbations in the pulse at the time $t=15$ is shown in Figs. 2, 3 and 4. Note, that in all the figures, pre-factor $\epsilon$ stands to underscore the fact that the linear problem, which we study, does not depend on the initial amplitude. Thus, we use $A=1$, while we have to bear in mind that linear approximation itself is valid for small amplitudes that is why we use small, arbitrary pre-factor $\epsilon$ in our notations.

A fairly good quantity describing the relation between different components of the pulse is the maximum of absolute value over the whole simulation domain. We plot this quantity for all physical variables in Fig. 5. In this figure, thick solid lines correspond to the resolution $256^{3}$, while thin solid lines to that of $128^{3}$, both for $\alpha_{y}=0.6$. It is remarkable that on all panels these two curves practically do overlap, which serves as a proof of convergence of our simulation results. The last figure in the bottom row presents the maximum of absolute value over the whole simulation domain of $\operatorname{div} \boldsymbol{B}$, and we indeed observe almost perfect fulfillment of the fundamental law, $\operatorname{div} \boldsymbol{B}=0$, which comes as a bonus of high-order, centered, finite difference numerical scheme. As expected, Fig. 5 shows that when $\alpha_{y}=0$, the pulse is perfectly Alfvénic (perturbing $V_{y}$ and $B_{y}$ only). This conclusion can be deducted either from analyzing Eqs. (11)-(13) or resorting to a classic mechanical analogy of coupled pendulums. In effect, Eqs. (11)-(13) also describe three inter-coupled mathematical pendulums which are located in $x O z$ plane. Thus, as long as we do not perturb these pendulums such that $\alpha_{y} \neq 0$, they will always oscillate in the $x O z$ plane. Thus, what we see in Fig. 5 when $\alpha_{y}=0$ (dashdotted lines) is that initial kinematic Alfvén perturbation $\left(V_{y}\right)$ is split in half-amplitude D'Alambert's solutions (both for $V_{y}$ and $B_{y}$ ) and no other physical quantity is generated. However, when we switch on the coupling, $\alpha_{y} \neq 0$, compressible perturbations are generated.

In order to investigate the effect of phase mixing on the created quasi-steady MHD state, we produced time series of all physical quantities in several points of the simulation 3D box, Figs. 7 and 8. Namely, at the points $(x=0, y=-8$, $z=0,4,8$ ), which are located in the phase mixing (spatial inhomogeneity) domain, and at ( $x=16, y=-8, z=0,4,8$ ), which are far away from it. The three panels (from the left to the right), in effect, trace the dynamics in the $z$-direction, i.e. along the regular magnetic field lines. These types of data would be obtained by, for example, three satellites which are located in three different regions of solar wind.

There are two noteworthy features that can be gathered from these plots: first, we see that in the phase mixing region, $V_{x}$, which is associated with the fast magnetosonic component of the pulse, attains about 40 times larger values than in the region that is far from the phase mixing region. That is a sensible result, since it is known that phase mixing efficiently generates oblique fast magnetosonic waves. Second, the total magnetic field perturbation $\left|\boldsymbol{B}-\boldsymbol{B}_{0}\right|$ is positively correlated with the pressure (as well as density, which is proportional to the gas pressure) perturbation, which indeed is what was expected from the fast magnetosonic wave.

Also, Figs. 5-8 demonstrate that, interacting with the plasma inhomogeneity, the longitudinally (parallel to the magnetic field) propagating part of the initial perturbation develops to an almost non-evolving (without change in amplitude) propagating state. In this quasi-steady MHD state all components of the pulse tend to propagate without change in amplitude, even though the effect of phase mixing is in action all the time ( $x$-component of the inverse characteristic spatial scale tends to infinity).

In Fig. 6 we investigate the energetics of our numerical simulation. In fact, we observe nearly perfect $( \pm 0.2 \%$ error $)$ conservation of total energy by $d t 4 d x 10$ numerical code. The major conclusion which can be drawn from this graph is that when $\alpha_{y}=0$ there is no internal (compressive) energy generation, while with the increase of $\alpha_{y}$ its final, asymptotic levels increase progressively.

The parametric space of the problem is studied in Fig. 9 by plotting the final levels of relative density perturbation, approached by the MHD pulse, as a function of the initial localization of the pulse in the $y$-direction $\alpha_{y}$, and plasma $\beta$. We gather from Fig. 9 that the achieved levels of density fluctuations depend weakly on $\alpha_{y}$, while there is somewhat stronger dependence on $\beta$.

\section{Conclusions}

In this paper we present results of numerical modeling of interaction of an initially localized MHD pulse with a transverse inhomogeneity of the plasma. The pulse is non-plane in all three 

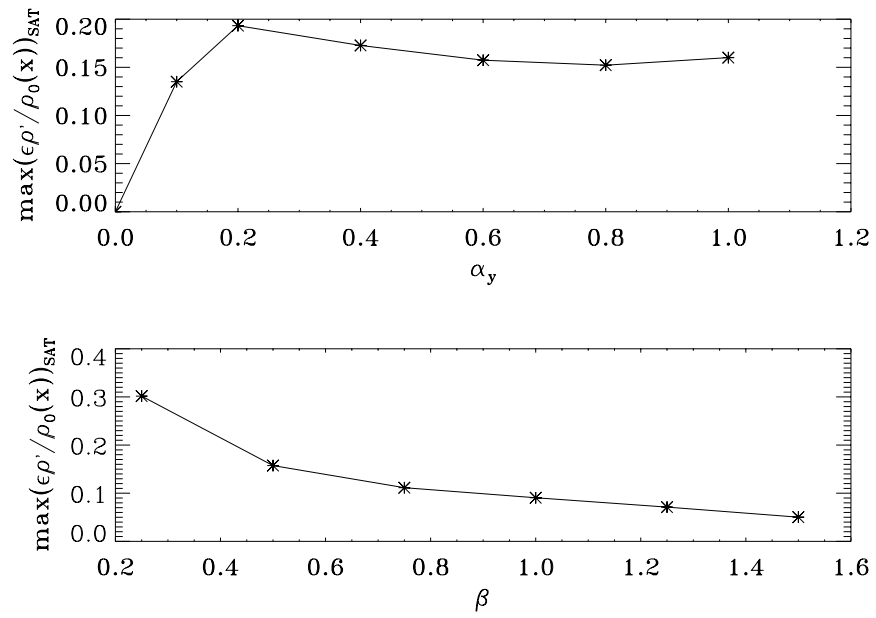

Fig. 9. Top panel: the final levels of relative density perturbations as a function of $\alpha_{y}$ (for $\beta=0.5$ and $\alpha_{z}=1.0$ ). Bottom panel: the same but as a function of $\beta$ (for $\alpha_{y}=0.6$ and $\alpha_{z}=1.0$ ).

spatial directions and is subject to the effect of phase mixing. An impulsively generated perturbation of the plasma velocity develops to an anisotropically propagating pulse, which has a significant compressible component. More specifically:

- The non-uniformness of the pulse in the homogeneous transverse (or, in other words, in the third, perpendicular to both the magnetic field and the inhomogeneity gradient) direction (non-zero $\alpha_{y}$ ) leads to appearance of compressive perturbations in the pulse.

- In the presence of plasma inhomogeneity, a non-uniform MHD pulse is essentially compressible. More specifically, when the pulse is initially plane in the homogeneous transverse direction, $\alpha_{y}=0$, there is no internal (compressive) energy generation, while with the increase of $\alpha_{y}$, the levels of the compressible energy increase progressively.

- A propagating MHD pulse asymptotically reaches a quasisteady state. The final levels of density perturbation, which can be considered as a measure of the compressibility in the pulse, depend weakly on $\alpha_{y}$, while there is somewhat stronger dependence on $\beta$.

- A smooth 1D transverse inhomogeneity of the plasma supports propagation of compressible MHD pulses. This mechanism of wave guiding is different from the wellunderstood phenomenon of refraction of fast magnetosonic waves, as the profile of the Alfvén speed in the inhomogeneity does not have a minimum.

- Weakly non-plane MHD waves are subject to phase mixing and can qualitatively be considered in terms of the $2.5 \mathrm{D}$ theory of Alfvén wave phase mixing. Consequently, the 2.5D theory of Alfvén wave phase mixing remains relevant to the 3D case too. However, quantitative theories of the interaction of MHD waves with plasma inhomogeneities should include the compressibility of the plasma as a necessary ingredient.

Our main conclusion is that the effects of threedimensionality, compressibility and inhomogeneity should be all together taken into account in the wave-based theories of coronal heating and solar wind acceleration, and as well as in the theories of MHD turbulence.

Acknowledgements. The authors are grateful to Tony Arber for valuable advise when $d t 4 d x 10$ code was written and for a number of valuable discussions. DT acknowledges financial support from PPARC. Numerical calculations of this work were performed using the PPARC funded Compaq MHD Cluster at St Andrews and Astro-Sun cluster at Warwick.

\section{References}

Botha, G. J. J., Arber, T. D., Nakariakov, V. M., \& Keenan, F. P. 2000, A\&A, 363, 1186

De Groof, A., \& Goossens, M. 2000, A\&A, 356, 724

DeMoortel, I., Hood, A. W., Ireland, J., \& Arber, T. D. 1999, A\&A, 346, 641

DeMoortel, I., Hood, A. W., \& Arber, T. D. 2000, A\&A, 354, 334

Génot, V., Louarn, P., \& Mottez, F. 2001, J. Geophys. Res., 106, 29633

Génot, V., Mottez, F., \& Louarn, P. 2000, J. Geophys. Res., 105, 27611

Génot, V., Louarn, P., \& Le Quéau, D. 1999, J. Geophys. Res., 104, 22649

Grappin, R., Léorat, J., \& Buttighoffer, A. 2000, A\&A, 362, 342

Heyvaerts, J., \& Priest, E. R. 1983, A\&A, 117, 220

Hood, A. W., Brooks, S. J., \& Wright, A. N. 2002, Proc. Roy. Soc. A (in press)

Hood, A. W., Ireland, J., \& Priest, E. R. 1997, A\&A, 318, 957

Hood, A. W., Gonzalez-Delgado, D., \& Ireland, J. 1997, A\&A, 324,11

Malara, F., Petkaki, P., \& Veltri, P. 2000, ApJ, 533, 523

Malara, F., Primavera, L., \& Veltri, P. 1996, ApJ, 459, 347

Nakariakov, V. M., Roberts, B., \& Murawski, K. 1997, Sol. Phys., 175, 93

Nakariakov, V. M., Roberts, B., \& Murawski, K. 1998, A\&A, 332, 795

Narain, U., \& Ulmschneider, P. 1996, Space Sci. Rev., 75, 453

Petkaki, P., Malara, F., \& Veltri, P. 1998, ApJ, 500, 483

Ruderman, M. S., Nakariakov, V. M., \& Roberts, B. 1998, A\&A, 338, 1118

Roussev, I., Galsgaard, K., Erdelyi, R., \& Doyle, J. G. 2001, A\&A, 370,298

Similon, P. L., \& Sudan, R. N. 1989, ApJ, 336, 442

Tsiklauri, D., Arber, T. D., \& Nakariakov, V. M. 2001, A\&A, 379, 1098

Tsiklauri, D., Nakariakov, V. M., \& Arber, T. D. 2002, A\&A, submitted 